\title{
SAMAR: Um Sistema Autônomo de Mensuração de Autorrelatos no auxílio à Formação de Grupos para o campo CSCL
}

Cícero Costa Quarto - Engenharia de Computação/UEMA - cicero@engcomp.uema.br Lucas de Souza Vieira - Engenharia de Computação/UEMA - lucaspakfa@ gmail.com

Magda Bercht - Instituto de Informática/UFRGS/PPGIE - bercht@inf.ufrgs.br Juliana Cerentini Pacico - Instituto de Psicologia/UFRGS - jucerentini@ hotmail.com Cláudio Simon Hutz - Instituto de Psicologia/UFRGS - claudio.hutz@ gmail.com

Resumo: Este artigo aborda a formação de grupos baseada no perfil de autorrelato do indivíduo, de forma a tornar a Aprendizagem Colaborativa Apoiada por Computador mais eficiente e assim auxiliar o professor na sua ação didática. O aporte teórico da pesquisa é alicerçado na Psicologia Positiva e na Computação. Da Computação, apresenta-se a ferramenta SAMAR, um sistema autônomo de mensuração de autorrelatos, implementado em PHP e integrado ao MySQL. Descreve-se um experimento no Ambiente Virtual de Aprendizagem MOODLE, através do qual se busca estudar a aplicação das variáveis de autorrelatos consideradas. Como principais resultados da pesquisa se destaca que os melhores desempenhos de grupos colaborativos foram alcançados naqueles grupos com perfis de autorrelatos de médio para alto e com formações de grupos heterogêneos.

Palavras-chave: CSCL. Formação de Grupos. Variáveis Psicológicas Positivas.

\section{SAMAR: An Autonomous Self-Reporting Measurement System in aid of Group Formation for CSCL Environments}

\begin{abstract}
This paper aims to discuss group formation based on individual's profile selfreport in order to make Computer Supported Collaborative Learning more efficient and thus helping teachers in their didactic actions. The theoretical contribution of the research is grounded in Positive Psychology and Computing. From Computing, the SAMAR tool is presented, an autonomous system of self-reporting measurement, implemented in PHP and integrated with MySQL. This paper also describes an experiment in Moodle Virtual Learning Environment, through which the application of the considered self-report variables was studied. The main results of the research stands out that the best performances of collaborative groups were reached in those groups with self-reported profiles from average to high and with heterogeneous groups formation
\end{abstract}

Keywords: CSCL. Group formation. Positive Psychological Variables.

\section{Introdução}

Os novos cenários que se apresentam hoje na educação online se utilizam dos Ambientes Virtuais de Aprendizagem (AVA) que são cada vez mais aliados a ferramentas tecnológicas de informação e comunicação, os quais vislumbram apoiar interação, 
cooperação, exigências diretamente relacionadas a uma perspectiva construtivista, reflexiva, colaborativa, que viabilize processos autônomos de aprendizagem, um dos quais a Aprendizagem Colaborativa Apoiada por Computador, a denominada CSCL (do inglês Computer Supported Collaborative Learning) (LOPES, 2007).

Wessner e Pfister (2001) constatavam que a aprendizagem colaborativa já tinha sido comprovada ser um método de sucesso em sala de aula tradicional, e que no campo da CSCL viria se tornar uma estratégia importante. Porém, ainda segundo estes autores, a maior parte dos cursos baseados na Web ainda aderiam à metodologia de aprendizagem individual, onde a comunicação e colaboração com outros alunos era geralmente arbitrário; na melhor das hipóteses, um AVA provia, em geral, ferramentas tais como sessões de batepapo ou Newsgroups para discussões dos conteúdos do curso.

Para Dillenbourg (2002), a eficácia da aprendizagem colaborativa depende de várias condições, dentre as quais a composição do grupo (tamanho, idade, sexo, heterogeneidade, ...), as características da tarefa (script ou roteiro) e dos meios de comunicação (síncrono ou assíncrono). Stahl et al. (2006), atentavam que com o suporte à colaboração entre os indivíduos em contextos de aprendizagem virtual, o campo CSCL tem fortes contribuições, mas também trouxe consigo alguns desafios, tais como: a) quais os perfis mais adequados dos alunos para a aprendizagem colaborativa? b) qual a melhor distribuição dos alunos em grupos, de forma a potencializar a aprendizagem colaborativa? e c) como modelar $e$ implementar computacionalmente tal abordagem? Corroborando Stahl et al. (2006), Graf e Bekele (2006) consideravam que representar a formação de grupos era um papel crucial para o sucesso de aprendizagem colaborativa. Conforme Abrami e Chambers (1996), através de experimentos pedagógicos, ambas estratégias de formação de grupos homogêneos e heterogêneos poderiam efetivamente promover colaboração.

A organização deste artigo é composta de seis seções, a partir da Seção 1 já descrita. Na Seção 2, apresenta-se uma introdução aos conceitos que apoiam esta pesquisa em Psicologia Positiva. A Seção 3 descreve a ferramenta AgIPA. Um experimento é descrito na Seção 4. Na Seção 5 são apresentados os Resultados do experimento. As Conclusões e trabalhos futuros são reservados para a Seção 6.

\section{Psicologia Positiva}

Conforme Seligman e Csikszentmihalyi (2000), Psicologia Positiva é a Ciência da experiência positiva subjetiva, traços individuais positivos e instituições positivas que procura melhorar a qualidade de vida e prevenir as patologias que surgem quando a vida é estéril e sem sentido. Ainda para os autores, a Psicologia Positiva busca explicar questões como o que permite que a felicidade, os efeitos da autonomia e auto-regulação, como o otimismo e esperança afetam a saúde e concluem que a Psicologia Positiva traz para este novo século contribuições para o entendimento científico e para intervenções em indivíduos, famílias e comunidades.

Baseado neste contexto, esta pesquisa explora instrumentos de avaliação em Psicologia Positiva já adaptados e/ou construídos e validados pelo Laboratório de Mensuração ${ }^{1}$, do Instituto de Psicologia da Universidade Federal do Rio Grande do Sul (UFRGS).

\footnotetext{
${ }^{1}$ http://www.ufrgs.br/psico-laboratorio
} 


\subsection{Variáveis Psicológicas Positivas}

Dentre as contribuições da Psicologia Positiva, destacam-se a construção de instrumentos de avaliação, modelos de intervenção e aplicação no curso desenvolvimental (PALUDO e KOLLER, 2007; SELIGMAN e CSIKSZENTMIHALYI, 2000). Seligman e Csikszentmihalyi (2000) listam como variáveis psicológicas positivas as que seguem: bemestar subjetivo, otimismo, autoestima, esperança, autoeficácia, dentre outras. Para Seligman (2004); Snyder et al. (2002); Day et al. (2010), as variáveis psicológicas positivas são usadas para medir os níveis individuais dos seres humanos, seu potencial pessoal e acadêmico.

Conforme Tierney (1995); Snyder et al. (2002); Bressler et al. (2011), a pontuação na escala de esperança prediz significativamente maiores GPAs (do inglês Grade Point Average), as taxas de graduação mais elevadas e menores taxas de evasão. Quanto maior for as pontuações de esperança, os estudantes terão mais inspiração e confiança, abordam problemas com foco no sucesso. Segundo Snyder et al. (2003), os baixos alunos em esperança podem dar-se quando são confrontados com desafio, principalmente porque eles não podem processar outras maneiras de triunfo sobre sua barreira. Além disso, a baixa esperança de estudante, quando confrontado com desafio, experimentam menos estresse associado com a tomada de teste (SNYDER et al., 2003).

Para Snyder et al. (2002); Simmons et al. (2003), pessoas com uma forte ênfase na esperança são mais dispostas a misturar-se com os colegas de grupo a quem eles não têm interesse comum, assim como se comunicam de forma positiva podendo ser valioso para os líderes em organizações, incluindo ambas as faculdades e empresas.

Seligman e Csikszentmihalyi (2000); Harrington (2002); Pacico e Bastianello (2014), chamam atenção que indivíduos que se percebem altamente eficazes ativam esforços suficientes, produzindo resultados excelentes e bem executados. Estes conseguem se automotivar, ter iniciativa, persistência e empenham esforços necessários porque acreditam que podem lidar com eventos de forma satisfatória. Ainda para estes autores, indivíduos que se percebem baixos em autoeficácia têm mais tendência a cessar seus esforços prematuramente e fracassar na execução das tarefas e concluem que a autoeficácia está relacionada a diversas variáveis, como por exemplo desempenho acadêmico.

Baumeister et al. (2003) atentavam que a autoestima causaria muitos resultados positivos em desempenho escolar e concluem que autoestima elevada facilitava a persistência após falhas. Alta autoestima torna as pessoas mais dispostas a falar em grupo e para criticar a abordagem do grupo. Liderança não decorre diretamente da autoestima, mas pode ter efeitos indiretos. Em relação a pessoas com baixa autoestima, aqueles com autoestima elevada mostram mais forte favoritismo em grupo. A alta autoestima leva a resultados mais felizes, independentemente de estresse ou outras circunstâncias. No geral, os benefícios da autoestima elevada se dividem em duas categorias: Iniciativa Reforçada e Sentimentos Agradáveis (BAUMEISTER et al., 2003).

A partir do contexto descrito nessa seção, constata-se que seus autores cientistaspesquisadores da Psicologia Positiva atentam fortemente a cautela para as variáveis psicológicas positivas esperança, autoeficácia e autoestima no impacto destas no 
desempenho educacional do indivíduo. Portanto, baseado nestas considerações, este trabalho descreve a ferramenta computacional SAMAR, capaz de mensurar o perfil de autorrelato do indivíduo baseado nas variáveis positivas citadas acima, de forma a auxiliar a formação otimizada de grupos para contextos CSCL. A seguir, define-se as variáveis psicológicas positivas citadas acima.

Esperança: Para Day et al. (2010); Bressler et al. (2011); Pacico e Bastianello (2014), esperança é definida como cognições voltadas para a obtenção de um objetivo, compostas por rotas e agenciamento. $\mathrm{O}$ agenciamento é a motivação do sujeito em perseguir a meta a ser alcançada, e as rotas são os caminhos planejados para obter tais objetivos. A esperança também pode ser identificada como uma estratégia cognitiva para atender e superar metas pessoais (GILLHAM, 2000; SNYDER, 1994); SNYDER et al. (2002) e KRAMER e CONOLEY (1992).

Autoeficácia: Segundo Bressler et al. (2011), autoeficácia envolve a confiança em alcançar metas pessoais/profissionais. Para Pacico et al. (2014), a crença na capacidade de realizar uma determinada tarefa, com base nos próprios recursos, foi definida por Bandura (1997) como autoeficácia. De acordo com Bandura (1997), a autoeficácia é a crença na capacidade de reunir recursos cognitivos, motivacionais e comportamentais necessários para a execução de uma tarefa que está no centro do conceito da autoeficácia (e não a realidade em si).

Autoestima: Para Baumeister et al. (2003); Hutz et al. (2014), a autoestima representa um aspecto avaliativo do autoconceito e consiste em um conjunto de pensamentos referentes a si mesmo. Trata-se, portanto, de uma orientação positiva (autoaprovação) ou negativa (depreciação) de se voltar para si mesmo e, nessa concepção, ela é a representação pessoal dos sentimentos gerais e comuns de autovalor.

Baseado no aporte teórico descrito nesta Seção, Na Figura 1, representa-se uma síntese da influência das variáveis psicológicas positivas Esperança, Autoestima e Autoeficácia nas áreas educacional (desempenho acadêmico, taxas de evasão, taxas de graduação, GPAs, motivação, proatividade, perspectivas positivas, humor positivo, crenças positivas, perseverança e habilidades e competências), Organizacional (motivação, perspectivas positivas, humor positivo, perseverança e habilidades e competências) e profissional (confiança, inspiração, tomada de decisões, motivação, perspectivas positivas, humor positivo, crenças positivas, empreendedorismo, perseverança e habilidades e competências). Acredita-se que esta representação venha contribuir rumo a direções futuras na construção de modelos do aluno para contextos CSCL, bem como no desenvolvimento de tecnologias para fins educacionais como Agentes Pedagógicos Afetivos e Sistemas Tutores Inteligentes (STI) considerando tais modelos afetivos do aluno. 


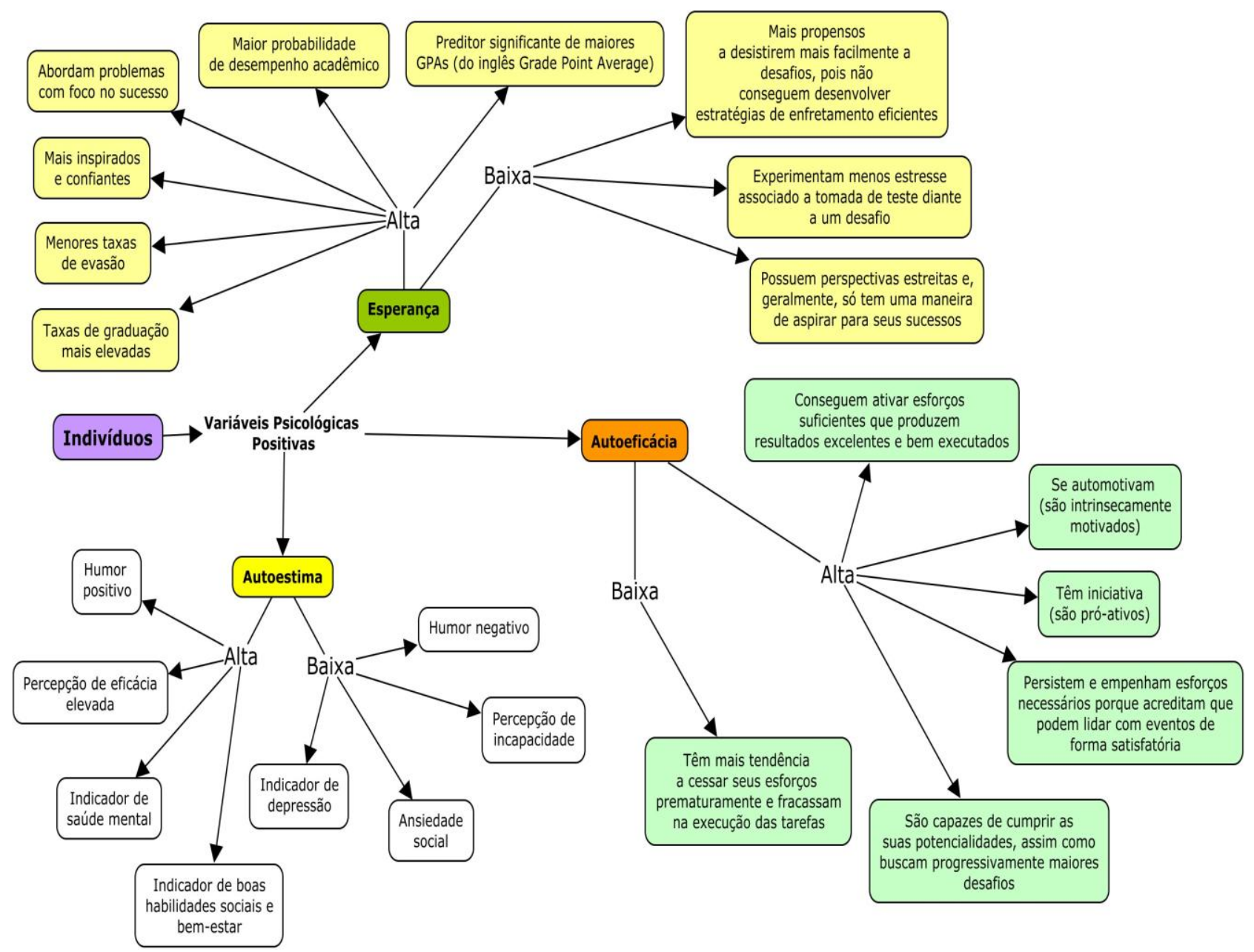

Figura 1: Influência das variáveis psicológicas positivas Esperança, Autoestima e Autoeficácia nas áreas Educacional, Organizacional e Profissional.

\subsection{Escalas de Autorrelatos}

Para Hutz et al. (2014), Escalas de Autorrelatos são instrumentos de avaliação para processo de coleta de informações, às quais auxiliam a conhecer e entender melhor o indivíduo. Escalas de Autorrelatos são uma forma válida e bastante utilizada para avaliar afetos $^{2}$. Nelas os participantes respondem o quanto têm experienciado emoções ou sentimentos ao longo de um período de tempo (HUTZ et al., 2014). Conforme Zanon e Hutz (2014), Escalas de Autorrelatos são compostas por conjuntos de itens, dispostos na forma de adjetivos ou sentenças, que descrevem aspectos positivos do indivíduo como emoções positivas ou negativas, a autoestima, o otimismo, a esperança e a autoeficácia.

Para as mensurações das variáveis psicológicas positivas Esperança (esp), Autoeficácia (aef) e Autoestima (aes) são utilizadas as escalas de Esperança Disposicional (Pacico e Bastianello, 2014), Escala de Autoeficácia Geral (Pacico et al., 2014) e a Escala de Autoestima de Rosenberg (Hutz et al., 2014). Todas essas escalas estão validadas pelo 
Instituto de Mensuração ${ }^{3}$, do Instituto de Psicologia, da Universidade Federal do Rio Grande do Sul (UFRGS). Na Tabela 1 são mostrados os níveis de perfis de autorrelatos do indivíduo em função das faixas de percentis ${ }^{4}$ considerando suas variáveis positivas Esperança, Autoeficácia e Autoestima. Esta classificação de níveis de autorrelatos foi definida junto ao Laboratório de Mensuração, do Instituto de Psicologia da UFRGS.

Tabela 1: Perfis de Autorrelatos Vs. Percentis

\begin{tabular}{c|c}
\hline \hline $\begin{array}{c}\text { Faixas de } \\
\text { Percentil }\end{array}$ & $\begin{array}{c}\text { Níveis de Autorrelatos do } \\
\text { indivíduo }\end{array}$ \\
\hline$<30 \%$ & Baixo (B) \\
\hline$\geq 30 \%$ e $<40 \%$ & Médio Baixo (MB) \\
\hline$\geq 40 \%$ e $<60 \%$ & Médio (M) \\
\hline$\geq 60 \%$ e $<70 \%$ & Médio Alto (MA) \\
\hline$\geq 70 \%$ & Alto (A) \\
\hline
\end{tabular}

\section{Descrição da ferramenta SAMAR}

A ferramenta SAMAR (Sistema Autônomo de Mensuração de Autorrelatos) foi desenvolvida com a finalidade de tornar a mensuração de Autorrelatos aos alunos de forma computacional. O SAMAR é uma ferramenta Web (Web Tool) que apresenta o TCLE (Termo de Consentimento Livre e Esclarecido) ao aluno, que tem a opção de participar ou não da pesquisa. Caso o participante aceite, ele é levado à uma seção de cadastro, onde são coletados dados básicos sobre o aluno, como nome, idade e sexo. Logo em seguida, o aluno é orientado a responder às três escalas de autorrelatos Esperança, Autoeficácia e Autoestima. A cada escala, o SAMAR armazena as respostas das questões apresentadas e mensura um autorrelato com base nas tabelas de cada escala, que é posteriormente armazenada em uma Base de Dados (BD). Ao responder todas as escalas, é mostrada ao aluno o seu perfil obtido em cada escala. A ferramenta SAMAR foi implementada usando PHP e uma base de dados relacional MySQL. Para efeito de ilustração das funcionalidades da ferramenta são mostradas nas Figuras 2, 3, 4 e 5, respectivamente, as interfaces Web do SAMAR para o TCLE, Cadastro do Participante e as Escalas de Esperança Disposicional e de Autoestima.

\footnotetext{
${ }^{2}$ Intensidade e a frequência com que as pessoas vivenciam emoções positivas e negativas [Zanon e Hutz, 2014].

3 http://www.ufrgs.br/psico-laboratorio/

4 Divide um conjunto de dados em 100 partes iguais $\mathrm{P}_{1}, \mathrm{P}_{2}, \mathrm{P}_{3}, \ldots, \mathrm{P}_{99}$. Usados para identificar valores excepcionalmente altos ou baixos. Por exemplo, as notas de um teste podem ser normalmente expressas em percentis. Notas no $95^{\circ}$ percentil ou acima são excepcionalmente altas, enquanto aquelas no $5^{\circ}$ percentil ou abaixo são excepcionalmente baixas (LARSON e FARBER, 2010).
} 


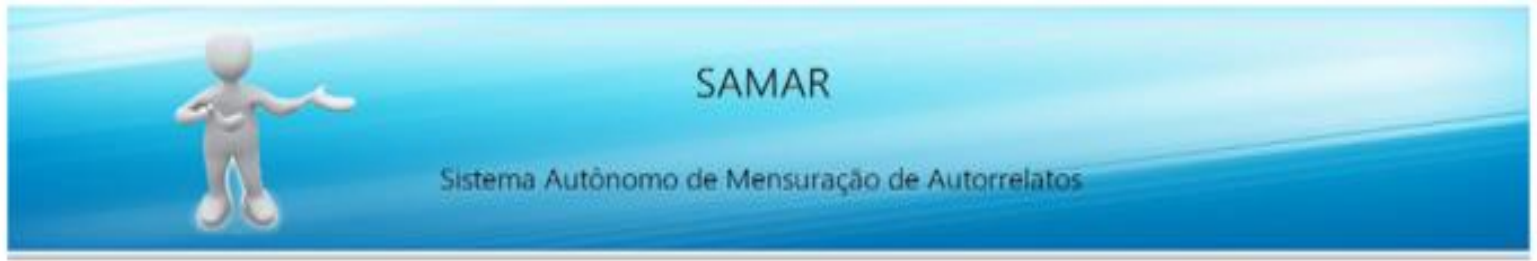

\section{Termo de Consentimento Livre e Esclarecido}

Estamos realizando um estudo de proposta de doutorado com o objetivo de investigar como potencializar a aprendizagem colaborativa apoiada por computador, atraves de indicadores positivos do individuo. Assim, voce esta sendo convidado(a) para realizar as atividades desse processo, em um formato a distância. A sua colaboração poderà contribuir para a construçăo do conhecimento cientifico e beneficiar perspectivas de intervençăa pedagógicas futuras. A participaçăo na pesquisa é totaimente voluntária. Esta pesquisa é coordenada pela Professora XXXXXXXXXXXXX(PPGIE/UFRGS) e pelo Doutorando XXXXXXXXXXXXXX do Programa de Pós-Graduaçlbo em Informática na Educaçào da Universidade Federal do Rio Grande do Sul (UFRGS) com quem podem ser obtidas maiores informaçées (Av. Paulo Gama, 110 - prédio 12105 - $3^{\circ}$ andar sala 332 CEP. 90040-060 - Porto Alegre RS - Brasil, e Laboratónio de Mensuraçăo da UFRGS - Rua Ramiro Barcelos, No 2600, Sala 101 - Bairro Santa Cecilia, Porto AlegreiRS - Brasii, )

Se vocé tiver dưvidas em relaçäo à pesquisa ou quiser comentar algum aspecto relacionado à mesma pode contatar os Pesquisadores responsàveis. A participaçăo na pesquisa e totalmente voluntảna. Portanto, caso nao queira participar vocé năo precisa assinar este termo nem participar da pesquisa. 0 fato de nào querer participar năo the nenhum prejuizo.

Após o encerramento do processo, voce pode solictar uma devolutiva individual. Os resultados globais da pesquisa serào publicados posteriormente em algum periodico ou evento cientifico da área de psicologia e informática na educaça, sem identficaçà da identidade dos participantes. Na apresentaçào dos resullados desse trabaiho, sua identidade será mantida no mais rigoroso sigilo. Serba omitidas todas as informaçōes que permitam identifica-lo(a)

Este documento fol revisado e aprovado pelo Comité de Ética da Universidade Federal do Rio Grande do Sul (nùmero CAAE 20469713.0.0000.5347)

Pelo presente Termo de Consentimento, eu declaro que sou maior de 18 anos, e que fui informado dos objetivos e da justificativa da presente pesquisa. E estou de acordo em participar da mesma. Fui igualmente informado. a) da liberdade de participar ou nắo da pesquisa, bem corno do meu direito de retirar meu consentimento, a qualquer momento, e deixar de participar do estudo, sem que isso me traga qualquer prejuizo, b) da garantia de receber resposta a qualquer dùvida acerca dos procedimentos e outros assuntos relacionados com a pesquisa, c) da segurança de que não seren identificado e de que se manterà o carăter confidencial das informaçöes registradas, d) que as informaçōes obtidas serä̀ armazenadas sem identificaçăo pessoal junto ao banco de dados do pesquisador responsável, e) que os dados da pesquisa serăo arquivados sob a quarda do pesquisador responsável por cinco anos e depois destruidos.

Pasicipar

Figura 2: Interface do TCLE do SAMAR.

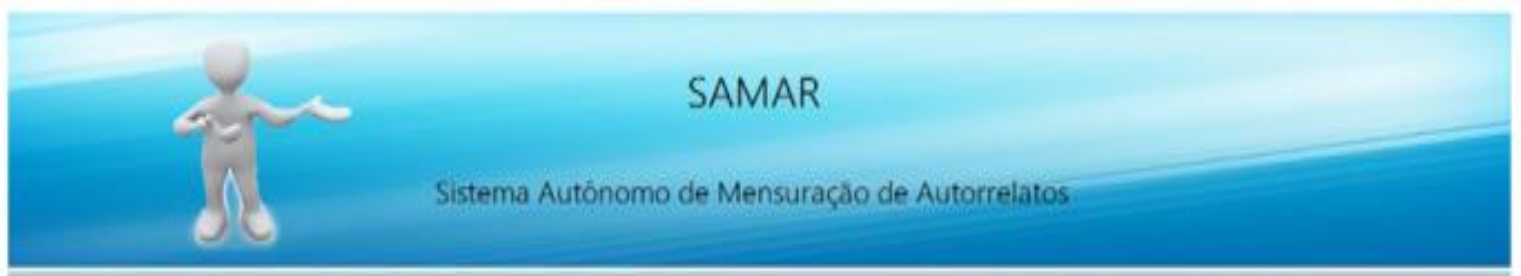

\section{Cadastro de Participante - SAMAR}

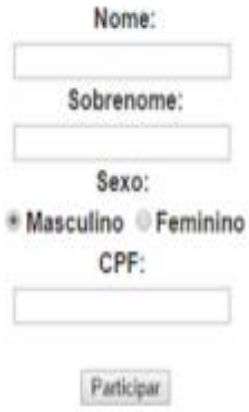

Figura 3: Interface de cadastro do SAMAR. 


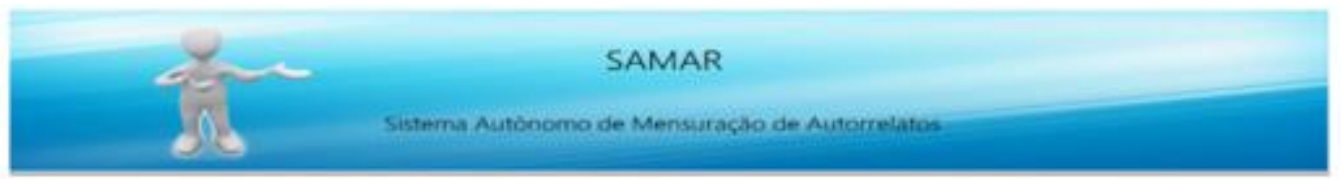

Esperança Disposicional

mestruçies

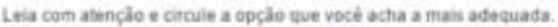

1. Es posso pensar en värias tormas de sdar con situaçbes eficeis.

Totalmente Falsa $01=2$ - $3=4$ * 5 Totalmecte Verdadein

2. Eume esforgo para atingr meus objetwos.

Totalmente Falsa $01020-3 \circ 4 * 5$ Totalnerte Verdadeina

3. Ee me simo cansado a maior parte do tempo.

Totalmente Falsa o 102 e 3 . 4 * 5 Totalmerte Verdadoine

4. Existem sempre muess tormas de resotver os protemas.

Totalmente Falsa o $1=2$ e 3 * 4 * 5 Totalmente Verdadeira

5. Eu sou tacimente derrobado em discussdes.

Totalmente Falsa $0102 \circ 3 \circ 4 \times 5$ Totalmecte Veedadeira

6. Ea posso pensar em muxas formas de conseguir as coisas qoe sho muho importantes para a minha vida.

Totalinente Falsa o 1 \& $2+3$ \& 4 \& 5 Tualmerte Vendadeira

7. Es me preocipo com a minha saise.

Totalmente Falsa o 1 : 2 e 3 * 4 * 5 Totalmente Verdadeita

c.Megmo guando os outros desistem, eu sei gie posso encentiar abuma forma de resolver os problemas.

Totalmente Falsa a $1=203$ * 4 * 5 Totalmente Verdadoira

2. Minhas experiéncias no passado me prepararam tem para eetrentar o fituro.

Totalmente Falsa $\circ 1 \circ 2 \circ 3 \circ 4 * 5$ Totalmente Verdadeira

10. Eu tenho tido muto sucesso na vida.

Totalmente Falsa $01+2 * 3 * 4=5$ Totalmente Verdadeira

11. Frequentemente es fico me preocupando con alouna colse.

Totalmente faisa o $102 * 3 \circ 4=5$ Totalnecese Verdadieta

12. Eu atino os objetivos que estabeleyo para mim

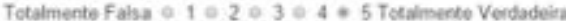

Figura 4: Interface de mensuração do SAMAR para o autorrelato Esperança Disposicional.

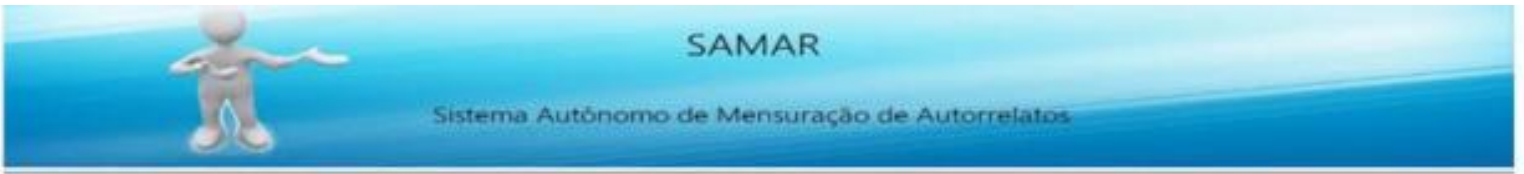

\section{Escala de Autoestima de Rosenberg}

Leia cada frase com atençăo e faça um circuio em torno da opçáo mais adequada

1) Eu sinto que sou uma pessoa de valor, no minimo tanto quanto as outras.

- Discordo totalmente - Discordo * Concordo e Concordo totalmente

2) Eu acho que eu tenho varuas boas qualidades.

- Discordo totaimente o Discordo Concordo * Concordo totalnente

3) Levando tudo em conta, eu penso que ou sou um fracasso.

- Discordo totalmente Discordo $e$ Concordo * Concordo totaimente

4) Eu acho que eu sou capaz de fazer as coisas tao bem quanto a maioria das pessoas.

- Discordo totalmente o Discordo * Concordo o Concordo totalmente

5) Eu acho que eu nào tenho muito do que me orgulhar.

- Discordo totaimente o Discordo + Concordo e Concordo totalmente

6) Eu tenho uma atitude positiva com relaça a mim mesmo.

- Discordo totalmente o Discordo e Concordo * Concordo totalmente

7) No conjunto, eu estou satisteito comigo.

- Discordo totalmente Discordo concordo + Concardo totaimente

8) Eu gostaria de poder ter mais respeito por mim mesmo.

- Discordo totaimente Discordo + Concordo e Concordo totaimente

9) As vezes eu me sinto inütil.

- Descordo iotaimento Oscordo + Concordo e Concordo totalmente

10) As vezes tu acho que eu nao presto para nada.

- Discordo totaimente * Discordo e Concordo e Concordo totalmente

Figura 5: Interface de mensuração do SAMAR para o autorrelato Autoestima. 


\section{Experimento}

O experimento foi baseado/instrumentalizado no AVA MOODLE do $\mathrm{CINTED}^{5} / \mathrm{UFRGS}$ e constituído pelas etapas Definição da amostra, Criação da sala virtual , Coleta de dados e mensuração de perfis de autorrelatos, Formação de grupos, Definição das atividades de aprendizagem e Avaliação da aprendizagem colaborativa. A seguir, descreve-se as referidas etapas: Definição da amostra: a amostra utilizada para o experimento foi a turma do primeiro período 2015.1 composta de trinta e seis alunos, do curso de Engenharia de Computação, da Universidade Estadual do Maranhão (UEMA). Criação da sala virtual: a sala virtual para o experimento foi criada no MOODLE e disponibilizada através da URL http://moodle2.cinted.ufrgs.br/. Coleta de dados e mensuração de perfis de autorrelatos: utilizando a ferramenta SAMAR, aplicou-se aos trinta e seis alunos o TCLE e as escalas de autorrelatos Esperança, Autoestima e Autoeficácia, de forma a mensurar os perfis de autorrelatos dos alunos baseados na Tabela 1. c) Formação dos grupos: para as formações de grupos, definiu-se as seguintes configurações: c.i) na Atividade 1 foram utilizados vinte e seis alunos distribuídos em seis grupos de alunos formados aleatoriamente (Grupo de Controle). Na Atividade 2 foram utilizados trinta e seis alunos distribuídos em nove grupos segundo critérios baseados nos perfis de autorrelatos dos alunos. d) Atividades de aprendizagem colaborativa: a Atividade 1 constituiu-se na produção textual pelos grupos, abrangendo conceitos da disciplina de Matemática Discreta Básica, composta dos itens: (i) elementos textuais (introdução, desenvolvimento e conclusões), (ii) elementos pós-textuais (referências, figuras, tabelas, apêndices e/ou anexos). A Avaliação 2 foi constituída de quarto questões descritivas abrangendo a Teoria dos Grafos. Para a avaliação de aprendizagem dos grupos convencionou-se duas variáveis: Pontuação Máxima Obtida na Atividade (PMOA) e o Tempo de Realização da Atividade (TRA). Estas variáveis foram usadas para avaliar $\boldsymbol{o}$ quão os grupos se empenharam na realização das atividades de aprendizagem, considerando para tal, os perfis de autorrelatos dos alunos que formaram cada grupo. O TRA foi estabelecido em duas horas, correspondendo a dois horários de hora/aula.

\section{Resultados}

Os resultados do experimento são apresentados e descritos a seguir através da Figura 6 e das Tabelas 2, 3 e 4.

\footnotetext{
${ }^{5}$ CINTED - Centro Interdisciplinar de Novas Tecnologias na Educação (http://www.cinted.ufrgs.br/)
} 


\section{Frequências de Perfis de Autorrelatos da Amostra de Alunos $(n=36)$}

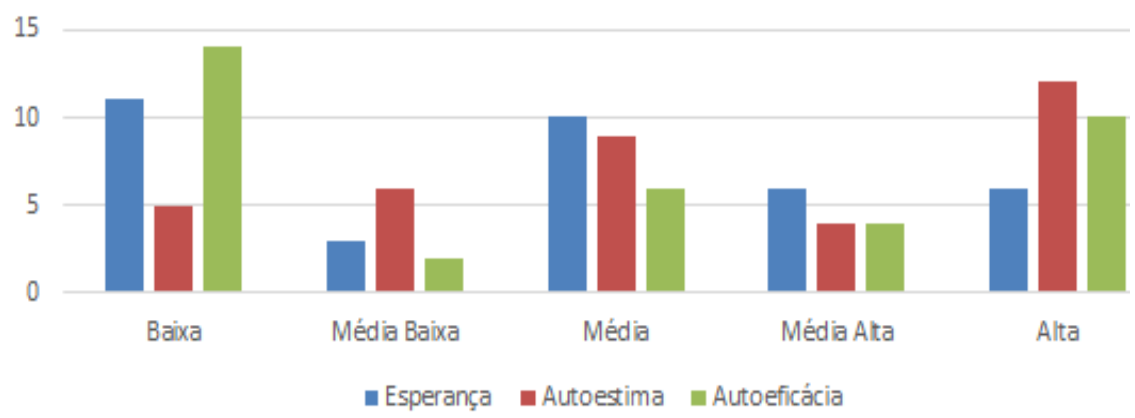

Figura 6: Caracterização da amostra Vs. Perfis de autorrelatos dos alunos.

O objetivo da Figura 6 é mostrar a frequência de cada nível de autorrelatos Esperança, Autoestima e Autoeficácia na amostra de alunos mensurados e ter a partir desta o mapeamento destes perfis, de forma que permitam formar grupos com várias configurações e assim ter condições de abstrair modelos de grupos para aprendizagem colaborativa. Pela Figura 5, observa-se que a amostra de alunos é bem heterogênea em relação aos níveis de autorrelatos Baixo, Médio Baixo, Médio, Médio Alto e Alto. Pode-se, também, observar que a amostra tem uma frequência baixa para o nível Baixo Médio.

Tabela 2: Perfis de autorrelatos da amostra.

\begin{tabular}{|c|c|c|c|c|c|c|c|c|c|c|c|c|c|c|c|c|}
\hline \multirow[b]{3}{*}{ Grupo } & \multirow[b]{3}{*}{ id_aluno } & \multicolumn{15}{|c|}{ PERFIS DE AUTORRELATOS } \\
\hline & & \multicolumn{5}{|c|}{ ESPERANÇA } & \multicolumn{5}{|c|}{ AUTOESTIMA } & \multicolumn{5}{|c|}{ AUTOEFICÁCIA } \\
\hline & & 1 & 2 & 3 & 4 & 5 & 1 & 2 & 3 & 4 & 5 & 1 & 2 & 3 & 4 & 5 \\
\hline \multirow{2}{*}{1} & Aln1_h_18a & & & $x$ & & & [ & & & & & $\diamond$ & & & & \\
\hline & Aln12_h_17a & & & $x$ & & & & & घ & & & & & & & 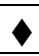 \\
\hline$\ldots$ & $\ldots$ & $\ldots$ & $\ldots$ & $\ldots$ & $\ldots$ & $\ldots$ & $\ldots$ & $\ldots$ & $\ldots$ & $\ldots$ & $\ldots$ & $\ldots$ & $\ldots$ & $\ldots$ & & $\ldots$ \\
\hline 3 & Aln15_h_17a & & & & & $x$ & & & & & - & & & & & $\checkmark$ \\
\hline 6 & Aln35_h_17a & & & $X$ & & & & & च & & & 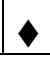 & & & & \\
\hline
\end{tabular}

Da Tabela 2, temos que Aln1_h_18a é o Aluno1, do Sexo Masculino (h) e idade de 18 anos, Aln31_m_17a é o Aluno31, do Sexo Feminino (m) e idade de 17 anos, e assim sucessivamente..., B - Baixa (1), MB - Média Baixa (2), M - Média (3), MA - Média Alta (4), A - Alta (5). Portanto, o Aluno1 tem um perfil de autorrelato do tipo Esperança Média (M0, Autoestima Baixa (B) e Autoeficácia Baixa (B). Este perfil é representado por uma tripla do tipo PerfA_Aln1 = \{esp: $B$, aes: $B$, aef: $B\}$. Para o Aluno15, temos que seu perfil autorrelato é PerfA_Aln15 = $\{$ esp: $A$, aes: $A$, aef: $A\}$ e assim sucessivamente.

Tabela 3: Resultados da Atividade 1 Vs. Perfis de Autorrelatos (Formação 1 de grupos).

\begin{tabular}{c|c|c|c|c|c|c}
\hline Perfis de autorrelatos & Grupo1 & $\begin{array}{c}\text { Grupo } \\
\mathbf{2}\end{array}$ & $\begin{array}{c}\text { Grupo } \\
\mathbf{3}\end{array}$ & $\begin{array}{c}\text { Grupo } \\
\mathbf{4}\end{array}$ & $\begin{array}{c}\text { Grupo } \\
\mathbf{5}\end{array}$ & $\begin{array}{c}\text { Grupo } \\
\mathbf{6}\end{array}$ \\
\hline esp_baixa & 0 & 1 & 1 & 2 & 1 & 2 \\
\hline$\ldots$ & $\ldots$ & $\ldots$ & $\ldots$ & $\ldots$ & $\ldots$ & $\ldots$
\end{tabular}




\begin{tabular}{c|c|c|c|c|c|c} 
esp_alta & 1 & 0 & 1 & 0 & 1 & 0 \\
aes_baixa & 1 & 0 & 0 & 0 & 1 & 1 \\
$\ldots$ & $\ldots$ & $\ldots$ & $\ldots$ & $\ldots$ & $\ldots$ & $\ldots$ \\
aes_alta & 0 & 1 & 3 & 1 & 2 & 2 \\
\hline aef_baixa & 2 & 1 & 0 & 2 & 2 & 2 \\
$\ldots$ & $\ldots$ & $\ldots$ & $\ldots$ & $\ldots$ & $\ldots$ & $\ldots$ \\
aef_alta & 1 & 0 & 2 & 0 & 1 & 2 \\
PMOA & 7,0 & 2,0 & 7,5 & 4,5 & 3,5 & 6,5 \\
TRA & 3 & 3 & 2 & 1 & 1 & 3 \\
\hline
\end{tabular}

Tabela 4: Resultados da Atividade 2 Vs. Perfis de autorrelato (Formação 2 de grupos).

\begin{tabular}{|c|c|c|c|c|c|c|c|c|c|c|c|c|c|c|c|c|c|}
\hline \multirow{3}{*}{ GRUPOS } & \multicolumn{15}{|c|}{ VARIÁVEIS PSICOLÓGICAS POSITIVAS } & \multirow{3}{*}{ PMOA } & \multirow{3}{*}{ TRA } \\
\hline & \multicolumn{5}{|c|}{ ESPERANÇA } & \multicolumn{5}{|c|}{ AUTOESTIMA } & \multicolumn{5}{|c|}{ AUTOEFICÁCIA } & & \\
\hline & B & $M B$ & $M$ & MA & $\mathrm{A}$ & $\mathrm{B}$ & $\mathrm{MB}$ & M & MA & A & B & $\mathrm{MB}$ & M & MA & A & & \\
\hline A & 0 & 0 & 0 & 0 & 4 & 0 & 0 & 0 & 0 & 0 & 0 & 0 & 0 & 0 & 0 & 9,0 & 2 \\
\hline $\mathrm{B}$ & 0 & 0 & 0 & 0 & 0 & 0 & 0 & 0 & 0 & 4 & 0 & 0 & 0 & 0 & 0 & 8,0 & 2 \\
\hline C & 0 & 0 & 0 & 0 & 0 & 0 & 0 & 0 & 0 & 0 & 0 & 0 & 0 & 0 & 4 & 9,5 & 1 \\
\hline $\mathrm{D}$ & 4 & 0 & 0 & 0 & 0 & 0 & 0 & 0 & 0 & 0 & 0 & 0 & 0 & 0 & 0 & 6,0 & 1 \\
\hline $\mathrm{E}$ & 0 & 0 & 0 & 0 & 0 & 3 & 1 & 0 & 0 & 0 & 0 & 0 & 0 & 0 & 0 & 9,5 & 1 \\
\hline $\mathrm{F}$ & 0 & 0 & 4 & 0 & 0 & 0 & 0 & 0 & 0 & 0 & 0 & 0 & 0 & 0 & 0 & 8,5 & 1 \\
\hline $\mathrm{G}$ & 0 & 0 & 0 & 0 & 0 & 0 & 0 & 0 & 0 & 0 & 4 & 0 & 0 & 0 & 0 & 9,0 & 1 \\
\hline $\mathrm{H}$ & 1 & 0 & 0 & 0 & 0 & 0 & 0 & 1 & 0 & 1 & 0 & 0 & 1 & 0 & 0 & 10,0 & 1 \\
\hline 1 & 0 & 0 & 0 & 1 & 0 & 0 & 1 & 0 & 0 & 0 & 0 & 0 & 0 & 0 & 1 & 9,0 & 2 \\
\hline
\end{tabular}

Da Tabela 3, observa-se que os melhores índices de PMOA e TRA foram observados nos grupos 1, 3 e 6, atentando que os perfis de autorrelatos destes grupos são de médio para alto nas três variáveis positivas, assim como a composição dos referidos grupos tem características heterogêneas. Outra característica observada nestes grupos foi que o TRA dos mesmos ficou em média acima do estipulado, podendo-se atribuir a isso um maior envolvimento dos membros dos grupos em articular estratégias nas soluções das tarefas de aprendizagem. Os menores índices de PMOA e TRA foram observados nos grupos 2, 4 e 5, atentando que os perfis de autorrelatos destes grupos são de baixo para médio nas três variáveis positivas, assim como a composição dos referido grupos tem características homogêneas. Outra característica observada nestes grupos foi que o TRA dos mesmos ficou em média abaixo do estabelecido, podendo-se atribuir a isso um baixo envolvimento dos membros dos grupos em articular estratégias nas soluções das tarefas de aprendizagem.

Da Tabela 4, observa-se que os grupos predominantemente homogêneos formados com perfis de autorrelatos altos tiveram rendimentos mais elevados, da mesma forma que os grupos com perfis de autorrelatos heterogêneos. Já o grupo $D$, formado predominantemente com perfis de autorrelato Esperança Baixa, teve um rendimento menor. 


\section{Conclusões e trabalhos futuros}

O referente estudo buscou contribuir com a sua investigação científica focado à frente das três grandes demandas para a CSCL "quais os perfis mais adequados dos alunos para a aprendizagem colaborativa?”, "qual a melhor distribuição dos alunos em grupos, de forma a potencializar a aprendizagem colaborativa?" e "como implementar computacionalmente tal abordagem?”. Em relação à primeira demanda, o trabalho, alicerçado em seu aporte teórico, contribui com a representação da influência de variáveis psicológicas positivas como esperança, autoestima e autoeficácia, de forma que esta representação do conhecimento, descrita através da Figura 1, sirva como norte na construção do modelo de aluno para contextos CSCL. Em relação à segunda demanda, os resultados indicaram que os melhores desempenhos de aprendizagem foram alcançados para os grupos com formações heterogêneas de perfis de autorrelatos esperança, autoestima e autoeficácia. Finalmente, em relação à terceira demanda da CSCL, o estudo apresentou a ferramenta SAMAR, capaz de mensurar o perfil de autorrelato do aluno e assim auxiliar na formação de grupos para contextos CSCL baseado em tais perfis.

Como trabalhos futuros, pode-se recomendar os seguintes: (i) modelar e implementar a ferramenta SAMAR orientado a agentes. (ii) dotar o SAMAR com afetividade, de forma a apoiar afetivamente o aluno, isto é, reconhecer e expressar emoções do aluno quando este estiver interagindo no sistema e assim poder motivá-lo ao longo de suas atividades de aprendizagem. (iii) aumentar os experimentos para testar uma maior quantidade de combinação de formação de grupos baseado nos perfis de autorrelatos esperança, autoeficácia e autoestima, e com isso poder mais eficazmente assegurar de que forma os perfis de autorrelatos influenciam a performance de trabalho de grupo em contextos CSCL.

\section{Referências}

BANDURA, A. "Self-efficacy: The exercise of control." (1997).

BAUMEISTER, R. F.; CAMPBELL, J. D.; KRUEGER, J. I.and VOHS, K. D. "Does high self-esteem cause better performance, interpersonal success, happiness, or healthier lifestyles?." Psychological science in the public interest 4.1 (2003): 1-44.

BRESSLER, L. A.; BRESSLER, M. S. and BRESSLER, M. E.. "Demographic and psychographic variables and the effect on online student success." Journal of Technology Research 2 (2011): 1-16.

DAY, L.; HANSON, K.; MALTBY, J.; PROCTOR, C. and WOOD, A. "Hope uniquely predicts objective academic achievement above intelligence, personality, and previous academic achievement." Journal of Research in Personality 44.4 (2010): 550-553.

DILLENBOURG, P. "Over-scripting CSCL: The risks of blending collaborative learning with instructional design." Three worlds of CSCL. Can we support CSCL? (2002): 61-91.

GRAF, S. e BEKELE, R. "Forming heterogeneous groups for intelligent collaborative learning systems with ant colony optimization." Intelligent Tutoring Systems. Springer Berlin Heidelberg, 2006.

GILLHAM, J. "The science of optimism and hope." The science of optimism and hope (2000). 
HUTZ, C. S. Avaliação em Psicologia Positiva / Organizador, Cláudio Simon Hutz. - Porto Alegre: Artmed, 2014.

HUTZ, C. S.; ZANON, C. e VAZQUEZ, A. C. S. A escala de autoestima de Rosenberg. In: Avaliação em Psicologia Positiva/Organizador, Cláudio Simon Hutz - Porto Alegre: Artmed, 2014.

KRAMER, J. e CONOLEY, J. "11th Mental Measurements Handbook." (1992).

LARSON, R. e FARBER, B. Estatística Aplicada. Quarta edição - São Paulo: Pearson Prentice Hall, 2010.

LOPES, M. S. S. "Avaliação da aprendizagem em atividades colaborativas em EaD viabilizada por um fórum categorizado." Rio de Janeiro 168 (2007).

PACICO, J. C. e BASTIANELLO, M. R. Instrumentos para avaliação da esperança: escala de esperança disposicional e escala de esperança cognitiva. In: Avaliação em Psicologia Positiva/Organizador, Cláudio Simon Hutz - Porto Alegre: Artmed, 2014.

PACICO, J. C.; FERRAZ, S. B. e HUTZ, C. S. Autoeficácia - Yes We Can! In: Avaliação em Psicologia Positiva/Organizador, Cláudio Simon Hutz - Porto Alegre: Artmed, 2014.

PALUDO, S. S. e KOLLER, H. Psicologia Positiva: uma nova abordagem para antigas questões. PAIDEIA, 17(36), 9-20, 2007.

SELIGMAN, M. e CSIKSZENTMIHALYI, M. Positive psychology: An introduction. American Psychologist, 55, 5-14, 2000.

SELIGMAN, M. EP. Authentic happiness: Using the new positive psychology to realize your potential for lasting fulfillment. Simon and Schuster, 2004.

SIMMONS, B.; NELSON, D. L. and QUICK, J. C. "Health for the hopeful: A study of attachment behavior in home health care nurses." International Journal of Stress Management 10.4 (2003): 361.

SNYDER, C. R. The psychology of hope: You can get there from here. Simon and Schuster, 1994.

SNYDER, C. R.; FELDMAN, D. B.; SHOREY, H. S. and RAND, K. L. "Hopeful choices: A school counselor's guide to hope theory." Journal of Personality and Social Psychology 65 (2002): 1061-1070.

SNYDER, C. R.; LOPEZ, S. J.; SHOREY, H. S.; RAND, K. L. and FELDMAN, D. B. "Hope theory, measurements, and applications to school psychology." School Psychology Quarterly 18.2 (2003): 122, 2003.

STAHL, G.; KOSCHMANN, T. and SUTHER, D. Computer-supported collaborative learning: An historical perspective. In R. K. Sawyer (Ed.), Cambridge handbook of the learning sciences (pp. 409-426). Cambridge, UK: Cambridge University Press, 2006.

TIERNEY, W. G. "Addressing Failure: Factors Affecting Native American College Student Retention." Journal of Navajo Education13.1 (1995): 3-7.

ZANON, C. e HUTZ, C. S. Afetos Positivos e Negativos: Definições, Avaliações e suas implicações para intervenções. In: Avaliação em Psicologia Positiva/Organizador, Cláudio Simon Hutz - Porto Alegre: Artmed, 2014.

WESSNER, M. e PFISTER, H. "Group formation in computer-supported collaborative learning." Proceedings of the 2001 international ACM SIGGROUP conference on supporting group work. ACM, 2001. 